\title{
Caracterização palinológica de espécies de Amaryllidaceae sensu stricto ocorrentes no nordeste brasileiro ${ }^{1}$
}

\author{
Anderson Alves-Araújo ${ }^{2,4}$, Francisco de Assis Ribeiro dos Santos ${ }^{3}$ e Marccus Alves ${ }^{2}$
}

Recebido em 2/10/2006. Aceito em 2/04/2007

\begin{abstract}
RESUMO - (Caracterização palinológica de espécies de Amaryllidaceae sensu stricto ocorrentes no nordeste brasileiro). Este trabalho teve com objetivo realizar as descrições polínicas de espécies nordestinas de Amaryllidaceae s.s. e Alliaceae e relacioná-las visando a sua delimitação taxonômica. Foram analisadas as espécies: Griffinia espiritensis Ravenna var. bahiana Preuss \& Meerow, Griffinia gardneriana (Herb.) Ravenna, Habranthus itaobinus Ravenna, Habranthus robustus Herb. ex Sweet, Habranthus sylvaticus Herb., Hippeastrum puniceum (Lam.) Kuntze, Hippeastrum stylosum Herb., Hymenocallis littoralis Salisbury Zephyranthes candida (Lindl.) Herb. e Nothoscordum pernambucanum Ravenna. As descrições foram feitas a partir da análise de grãos de pólen acetolisados (ou não) observados em microscopia óptica e eletrônica de varredura, os quais foram obtidos de espécimes recém-coletados. Todas as espécies apresentaram grãos de pólen em mônades, heteropolar-bilaterais, monossulcados e âmbito elíptico/elipsoidal. Foram detectadas características diagnósticas com relação ao padrão de ornamentação, destacando Hippeastrum stylosum Herb. e Hymenocallis littoralis Salisbury por possuírem grãos de pólen com calotas equatoriais. As características polínicas analisadas foram suficientemente relevantes para a delimitação específica dentre os táxons analisados.
\end{abstract}

Palavras-chave: Amaryllidaceae, Alliaceae, Palinologia, Taxonomia, Brasil

\begin{abstract}
Pollen characterization of Amaryllidaceae sensu stricto species from northeastern Brazil). The pollen of Amaryllidaceae s.s. and Alliaceae species from northeastern Brazil is described and related to taxonomic delimitation. The species analyzed were: Griffinia espiritensis Ravenna var. bahiana Preuss \& Meerow, Griffinia gardneriana (Herb.) Ravenna, Habranthus itaobinus Ravenna, Habranthus robustus Herb. ex Sweet, Habranthus sylvaticus Herb., Hippeastrum puniceum (Lam.) Kuntze, Hippeastrum stylosum Herb., Hymenocallis littoralis Salisbury, Zephyranthes candida (Lindl.) Herb. and Nothoscordum pernambucanum Ravenna. Descriptions of acetolyzed and non-treated pollen grains from fresh specimens were based on analysis in light and scanning electron microscopy. All species presented pollen grains in heteropolar and bilateral, monossulcate and elliptic/ellipsoidal monads. Exine sculpture patterns showed many divergent features; Hippeastrum stylosum Herb. and Hymenocallis littoralis Salisbury had pollen grains with equatorial calotas where exine ornamentation is different. The pollen features analyzed were relevant to species delimitation among the taxons.
\end{abstract}

Key words: Amaryllidaceae, Alliaceae, Palynology, Taxonomy, Brazil

\section{Introdução}

A família Amaryllidaceae é amplamente distribuída pelo globo com grande parte de seus representantes nas regiões tropicais e subtropicais (Meerow 2004). No Brasil é representada principalmente pelos gêneros Habranthus Herb. e Hippeastrum Herb., além de apresentar táxons endêmicos como Griffinia Ker Gawl. e Worsleya Traub (Meerow \& Snijman 1998).

Estudos palinológicos envolvendo espécies de Amaryllidaceae são raros e, geralmente, fazem parte de catálogos e/ou levantamentos polínicos. No trabalho desenvolvido por Erdtman (1966), o autor ilustrou a morfologia polínica de vários táxons distribuídos dentre diversas famílias de angiospermas, incluindo cerca de 90 espécies distribuídas em 60 gêneros dos representantes classificados como Amaryllidaceae. Da mesma forma, Sharma (1967), Huang (1972) e Rao \& Ling (1974) analisaram a estrutura dos grãos de pólen de espécies tropicais asiáticas dessa família.

Dentre os gêneros polinicamente observados, os mais comuns nos levantamentos são Crinum L. (Hassal

Parte da Dissertação do primeiro Autor

2 Universidade Federal de Pernambuco, Centro de Ciências Biológicas, Departamento de Botânica, Laboratório de Morfo-Taxonomia Vegetal, Rua Professor Morais Rego s.n., Cidade Universitária, 50670-901 Recife, PE, Brasil

3 Universidade Estadual de Feira de Santana, Departamento de Ciências Biológicas, Laboratório de Micromorfologia Vegetal, Av. Universitária s.n., Campus Universitário, 44031-460 Feira de Santana, BA, Brasil

4 Autor para correspondência: agalves_araujo@hotmail.com 
1842; Erdtman 1966; Sharma 1967; Huang 1972), Hymenocallis Salisb. (Huang 1972; Meerow \& Dehgan 1985) e Zephyranthes Herb. (Sharma 1967; Rao \& Ling 1974).

Um dos mais importantes estudos palinológicos que pode ser citado para monocotiledôneas é o desenvolvido por Zavada (1983), no qual são comparados os grãos de pólen de representantes de várias ordens e sugeridas prováveis linhas evolutivas relacionadas aos tipos de aberturas, estrutura e ornamentação da exina. Tais caracteres já eram discutidos por Hassal (1842) quanto à sua utilização e importância para classificação de grupos de plantas.

Com espécies de Amaryllidaceae sul-americanas, poucos são os trabalhos investigativos com relação à morfologia polínica. Meerow \& Dehgan (1985) analisaram os grãos de pólen de Hymenocallis quitoensis Herb. quanto à estrutura da exina e suas implicações taxonômicas. Meerow et al. (1986), apresentaram tanto dados relacionados à palinologia de Stenomesson elwesii (Baker) Macbr. quanto à dispersão dos grãos de pólen. Meerow \& Dehgan (1988) estudaram o pólen de espécies pertencentes à Tribo Eucharideae ocorrentes na região amazônica, e Meerow (1989), que além de verificar a morfologia polínica, analisou diversos caracteres morfológicos e anatômicos de espécies dos gêneros Eucharis Planch., Caliphruria Herb. e Urceolina Rchb.

Face à grande lacuna existente no conhecimento palinológico das Amaryllidaceae brasileiras e considerando seu efetivo potencial para uso em estudos taxonômicos e filogenéticos, os objetivos do presente trabalho são descrever e relacionar a morfologia polínica de espécies da família ocorrentes no nordeste brasileiro como ferramenta para a delimitação taxonômica das mesmas.

\section{Materiais e métodos}

Segundo a proposta sugerida pelo APG II (2003), Alliaceae s.l. é constituída por Agapanthaceae, Amaryllidaceae s.s. e Alliaceae s.s. Entretanto, para melhor entendimento sistemático, os táxons aqui estudados serão considerados como Amaryllidaceae s.s., sendo Nothoscordum pernambucanum Rav. (Alliaceae s.s.) incluída por ser um táxon nativo da região e empregado para subsidiar abordagens comparativas.

O material polínico analisado foi proveniente de flores frescas das espécies Griffinia espiritensis Ravenna var. bahiana Preuss \& Meerow, Griffinia gardneriana (Herb.) Ravenna, Habranthus itaobinus Ravenna, Habranthus robustus Herb. ex Sweet, Habranthus sylvaticus Herb., Hippeastrum puniceum (Lam.) Kuntze, Hippeastrum stylosum Herb., Hymenocallis littoralis Salisb., Zephyranthes candida (Lindl.) Herb. e Nothoscordum pernambucanum Ravenna.

As exsicatas foram depositadas no Herbário Geraldo Mariz - UFP da Universidade Federal de Pernambuco e duplicatas cedidas aos herbários HUEFS, IPA, R, RB, SPF, UEC e UPCB, siglas de acordo com Holmgren et al. (2006). A coleta do material botânico priorizou os municípios do Estado de Pernambuco; entretanto foram incluídas espécies distribuídas pelo Nordeste brasileiro, cujos locais e números de coleta estão disponíveis na Tab. 1 .

Os grãos de pólen foram obtidos de anteras recémcoletadas armazenadas em ácido acético glacial PA e, posteriormente, submetidos à técnica padrão de acetólise segundo Erdtman (1960).

Para análise em microscopia óptica (M.O.), as lâminas foram montadas em gelatina glicerinada, a partir das quais foram tomadas as medidas dos diâmetros polar, equatorial maior e menor, aleatoriamente, de 25 grãos de pólen (sempre que possível), enquanto que para mensuração da espessura da exina foram empregadas dez unidades poliníferas. Para definição da forma, utilizou-se para o cálculo do $\mathrm{P} / \mathrm{E}$, o diâmetro equatorial maior.

Após a acetólise, o sedimento polínico foi submetido à série etílica crescente (30-100\%) e, em seguida, gotejado diretamente sobre o porta-espécimen do microscópio eletrônico de varredura (MEV). Depois de completa evaporação do etanol e secagem, a preparação foi metalizada com ouro-paládio (Metalizador SCD 050) e eletromicrografados sob MEV (LEO 1430 VP). Também foram obtidas fotomicrografias digitais em microscópio Zeiss (Axioskope 2).

As lâminas foram depositadas na Palinoteca do Laboratório de Micromorfologia Vegetal - LAMIV da Universidade Estadual de Feira de Santana - UEFS e a nomenclatura polínica adotada seguiu o sugerido por Punt et al. (1994).

\section{Resultados e discussão}

Os grãos de pólen das espécies estudadas de Amaryllidaceae s.s. e Alliaceae s.s. apresentaram características compatíveis com os dados publicados pelos autores consultados. Todos os táxons possuem 
Tabela 1. Caracteres morfométricos dos grãos de pólen de espécies de Amaryllidaceae s.s. e Nothoscordum (Alliaceae s.s.) nativas do Nordeste brasileiro.

\begin{tabular}{|c|c|c|c|c|c|c|}
\hline \multirow[t]{2}{*}{ Espécie/Espécimens } & \multirow[b]{2}{*}{ Diâmetro Polar* } & \multicolumn{2}{|c|}{ Diâmetro equatorial* } & \multirow[b]{2}{*}{$\mathrm{P} / \mathrm{E}$} & \multirow[b]{2}{*}{ Exina* } & \multirow[b]{2}{*}{$\mathrm{S}: \mathrm{N}$} \\
\hline & & Maior & Menor & & & \\
\hline \multicolumn{7}{|l|}{ Griffinia espiritensis Rav. var. bahiana Preuss \& Meerow } \\
\hline A. Alves-Araújo et al. 37 (Cabo Sto Agostinho-PE) & (25) $31(37)$ & $(50) 56(67)$ & (20) $28(35)$ & 0,56 & $1(2)$ & $\mathrm{S}=\mathrm{N}$ \\
\hline A. Alves-Araújo et al. 37 (a)** & $(27) 31(37) \dagger$ & $(50) 63(70)$ & (20) $30(35)$ & 0,50 & 1 & $\mathrm{~S}=\mathrm{N}$ \\
\hline A. Alves-Araújo et al. 37 (b)** & (23) $30(37)$ & (47) $55(65)$ & (23) $29(35)$ & 0,54 & $1(2)$ & $\mathrm{S}=\mathrm{N}$ \\
\hline \multicolumn{7}{|l|}{ Griffinia gardneriana (Herb.) Rav. } \\
\hline A. Alves-Araújo et al. 33 (Buíque - PE) & (40) $45(50)$ & (83) $89(113)$ & (25) $47(63)$ & 0.45 & 1 & $\mathrm{~S}=\mathrm{N}$ \\
\hline A. Alves-Araújo et al. 35 (Buíque-PE) & (30) $41(50)$ & (77) $87(100)$ & (20) $33(50)$ & 0,46 & 1 & $\mathrm{~S}=\mathrm{N}$ \\
\hline A. Alves-Araújo et al. 53 (Buíque - PE) & (30) $37(45)$ & (75) $81(105)$ & (27) $38(50)$ & 0,44 & 1 & $\mathrm{~S}=\mathrm{N}$ \\
\hline \multicolumn{7}{|l|}{ Habranthus itaobinus Rav. } \\
\hline A. Alves-Araújo et al. 22 (Agrestina-PE) & $(25) 33(45) \dagger$ & (53) $71(80)$ & (25) $35(50)$ & 0,48 & $1-2$ & $\mathrm{~S}=\mathrm{N}$ \\
\hline A. Alves-Araújo et al. 36 (Bezerros - PE) & $(25) 32(37)$ & $(45) 59(75)$ & (20) $28(37)$ & 0,53 & 1 & $\mathrm{~S}=\mathrm{N}$ \\
\hline A. Alves-Araújo et al. 50 (Bezerros-PE) & (25) $31(40)$ & $(50) 61(67)$ & (20) $28(35)$ & 0,51 & 1 & $\mathrm{~S}=\mathrm{N}$ \\
\hline \multicolumn{7}{|l|}{ Habranthus robustus Herb. ex Sweet } \\
\hline A. Alves-Araújo et al. 43 (em cultivo - BA) & (23) $31(35)$ & $(55) 62(65)$ & (23) 28 (37) & 0,49 & 2 & $\mathrm{~S}>\mathrm{N}$ \\
\hline A. Alves-Araújo et al. 43 (a)** & (25) $31(37)$ & $(53) 60(65)$ & (23) $28(35)$ & 0,51 & $(1) 2$ & $\mathrm{~S}>\mathrm{N}$ \\
\hline A. Alves-Araújo et al. 43 (b)** & (23) 30 (37) & $(55) 61(67)$ & (25) $31(40)$ & 0,48 & 2 & $\mathrm{~S}>\mathrm{N}$ \\
\hline \multicolumn{7}{|l|}{ Habranthus sylvaticus Herb. } \\
\hline A. Alves-Araújo et al. 30 (Buíque - PE) & (23) $30(35)$ & $(60) 72(80)$ & (25) $35(45)$ & 0,41 & 2 & $\mathrm{~S}=\mathrm{N}$ \\
\hline A. Alves-Araújo et al. 31 (Buíque - PE) & (27) $33(45)$ & $(50) 66(85)$ & (17) $30(50)$ & 0,51 & $(1) 2$ & $\mathrm{~S}=\mathrm{N}$ \\
\hline A. Alves-Araújo et al. 44 (Feira de Santana-BA) & (27) $33(40)$ & (47) $57(65)$ & $(25) 30(35)$ & 0,57 & (1) 2 & $\mathrm{~S}=\mathrm{N}$ \\
\hline \multicolumn{7}{|l|}{ Hippeastrum puniceum (Lam.) Kuntze } \\
\hline A. Alves-Araújo et al. 40 (Bezerros-PE) & $(30) 40(50)$ & (77) $87(100)$ & (33) $39(57)$ & 0,45 & 3 & $\mathrm{~S}>\mathrm{N}$ \\
\hline A. Alves-Araújo et al. 40 (a)** & (33) 43 (47) & (83) $93(117)$ & (33) $43(53)$ & 0,42 & $(2) 3$ & $\mathrm{~S}>\mathrm{N}$ \\
\hline A. Alves-Araújo et al. 49 (São Lourenço da Mata-PE) & $(27) 39(50)$ & (83) $102(123)$ & $(33) 53(70)$ & 0,34 & $(2) 3$ & $\mathrm{~S}>\mathrm{N}$ \\
\hline \multicolumn{7}{|l|}{ Hippeastrum stylosum Herb. } \\
\hline A. Alves-Araújo et al. 39 (Pombos - PE) & (33) $41(55)$ & (77) $94(120)$ & $(25) 40(50)$ & 0,40 & $2(3)$ & $\mathrm{Se}^{\prime} \mathrm{N}$ \\
\hline A. Alves-Araújo et al. 47 (Cabo Sto Agostinho-PE) & (33) $38(47)$ & (63) $78(100)$ & (27) $43(70)$ & 0,45 & (1) 2 & $\mathrm{~S}>\mathrm{N}$ \\
\hline A. Alves-Araújo et al. 51 (Bezerros - PE) & (33) 39 (47) & (83) $96(103)$ & (30) $41(50)$ & 0,38 & (1) 2 & $\mathrm{~S}>\mathrm{N}$ \\
\hline \multicolumn{7}{|l|}{ Hymenocallis littoralis Salisb.s } \\
\hline A. Alves-Araújo et al. 54 (Ipojuca-PE) & (80) $96(127)$ & (155) $169(190)$ & (85) $109(135)$ & 0,51 & (2)11(12) & $\mathrm{S}>\mathrm{N}$ \\
\hline A. Alves-Araújo et al. 54 (a)** & (75) $98(117)$ & (153) $169(210)$ & (75) $97(123)$ & 0,53 & (2) $10(11)$ & $\mathrm{S}>\mathrm{N}$ \\
\hline A. Alves-Araújo et al. 54 (b) ${ }^{* *}$ & $(85) 99(125)$ & (157) $171(185)$ & (77) $96(133)$ & 0,53 & (2) $10(11)$ & $\mathrm{S}>\mathrm{N}$ \\
\hline \multicolumn{7}{|l|}{ Zephyranthes candida (Lindl.) Herb. } \\
\hline A. Alves-Araújo et al. 38 (em cultivo-PE) & (27) $33(37)$ & $(47) 56(65)$ & $(30) 34(40) \dagger$ & 0,58 & $(1) 2$ & $\mathrm{~S}=\mathrm{N}$ \\
\hline \multicolumn{7}{|l|}{ Nothoscordum pernambucanum Rav. (Alliaceae s.s.) } \\
\hline A. Alves-Araújo et al. 76 (Pombos - PE) & (13) $17(23)$ & $(35) 40(45)$ & (15) $24(27)$ & 0,39 & 1 & $\mathrm{~S}=\mathrm{N}$ \\
\hline A. Alves-Araújo et al. $76(\mathrm{a})^{* *}$ & (15) $21(27)$ & (35) $41(47)$ & (20) $24(30)$ & 0,47 & 1 & $\mathrm{~S}=\mathrm{N}$ \\
\hline
\end{tabular}

* - média (faixa de variação), medidas em mm; ** - Ind. clones; $\uparrow$ - $\mathrm{n}<25$; P/E - razão entre os diâmetros polar/equatorial maior; S: - relação entre a espessura da sexina e da nexina.

grãos de pólen em mônades, heteropolares, bilaterais e monossulcados. As interpretações dos caracteres morfológicos das espécies estudadas seguem abaixo, bem como seus dados morfométricos encontram-se na Tab. 1.

\section{Griffinia Ker Gawler}

\section{Fig. 1-5}

Grãos de pólen grandes (a muito grandes), peroblatos a oblatos, amb elipsoidal. Os sulcos são tão longos quanto os diâmetros equatoriais maiores e com margens irregulares. Exina fina (ca. $1 \mathrm{~mm}$ de espessura) e com teto psilado (sob M.O.); entretanto, sob MEV (Fig. 3-5), podem ser observadas perfurações e processos supra-tectais baculiformes com extremidades arredondadas. As columelas são pequenas e finas; sexina e nexina de espessura semelhante.

Os dois táxons de Griffinia Ker Gawl. analisados são semelhantes palinologicamente, contudo os grãos de pólen de Griffinia espiritensis Ravenna var. bahiana Preuss \& Meerow (Fig. 1-2) são menores 
quando comparados aos de Griffinia gardneriana (Herb.) Ravenna (Fig. 3, Tab. 1). Essa espécie apresenta ainda processos baculiformes supra-tectais (Fig. 4-5); contudo ressaltamos que Griffinia espiritensis Rav. var. bahiana Preuss \& Meerow não teve seus grãos de pólen analisados sob MEV e assim poderá também apresentar iguais processos supratectais em seus grãos de pólen. Destacamos ainda que o teto fino e transparente dos grãos de pólen pode levar ao examinador ao falso diagnóstico da ornamentação, pois ele exibe - por transparência - a estrutura columelar interna à parede e não algo superficial.

Preuss (1999), ao estudar Griffinia Ker Gawl., abordou dados morfométricos, ecológicos e cariológicos. Porém, caracteres da morfologia polínica não foram informados, embora tenha sido mencionada a coloração dos grãos de pólen (Preuss 2004). A coloração observada pelo autor foi considerada uma característica distintiva entre Griffinia subgen. Griffinia Rav. e Griffinia subgen. Hyline (Herb.) Rav., que apresentaram as cores alva e amarela, respectivamente. Posteriormente, Preuss \& Meerow (2001), analisando Griffinia espiritensis ( $G$. subgen. Griffinia), verificaram diferentes faixas de coloração dos grãos de pólen entre suas variedades. No entanto, a abordagem dada à variação na coloração do pólen não é convincente, principalmente, devido às lacunas deixadas quanto à interpretação de diferentes matizes das cores, uma vez que, todas são derivadas da inicial alva (Preuss 1999).

Quanto à ornamentação da exina, os grãos de pólen de Griffinia gardneriana (Herb.) Ravenna são muito semelhantes ao descrito por Erdtman (1966) e Zavada (1983) para Crinum americanum L., que segundo os autores, apresentam espículos e, neste caso, podem ser comparáveis aos processos baculiformes observados no táxon aqui tratado, embora os táxons em questão não estejam diretamente relacionados filogeneticamente.

\section{Habranthus Herb.}

Fig. 6-9

Grãos de pólen grandes, peroblatos a oblatos, amb elipsoidal. Os sulcos são tão longos quanto os diâmetros equatoriais maiores e com margens irregulares. Exina fina $(1-2 \mu \mathrm{m})$, reticulada, homo a heterobrocada, columelas pequenas e finas. Sob MEV, muitas áreas da exina de Habranthus sylvaticus Herb. apresentaram-se com o retículo quase que retipilada, no qual os pilos apresentaram uma tênue ligação tectal entre si (Fig. 9). Sexina e nexina são semelhantes em espessura podendo, contudo, a primeira ser mais espessa.

A presença de exina reticulada é comum às amostras do gênero estudadas; destacamos que nas áreas em que a exina aparece retipilada (Fig. 9) a cabeça dos pilos são de diferentes tamanhos e muita vezes não fusionadas por completo. As espécies Habranthus sylvaticus Herb. e Habranthus itaobinus Ravenna são distintas pela presença de um microrretículo heterobrocado na primeira e homobrocado na segunda. Contudo, em Habranthus robustus Herb. ex Sweet o microrretículo apresentase com lumens maiores (Fig. 8-9) e a sexina é mais espessa em relação à nexina quando comparado às outras espécies analisadas (Tab. 1).

A ornamentação dos grãos de pólen de Habranthus Herb. não difere significativamente dos táxons que já foram analisados dentre vários representantes de Amaryllidaceae. O padrão reticulado é muito difundido, sendo constatado nos gêneros Crinum L. (Huang 1972; Erdtman 1966), Eucharis Planch. (Meerow \& Dehgan 1988), Haemanthus L. (Sharma 1967), Hymenocallis Salisb. (Huang 1972; Erdtman 1966), Lycoris Herb. (Huang 1972), Narcissus L. (Huang 1972), dentre outros. Esta é a primeira referência palinológica para espécies de Habranthus Herb.

\section{Hippeastrum Herb.}

Fig. 10-13

Grãos de pólen grandes (a muito grandes), peroblatos, amb elipsoidal. Os sulcos são tão longos quanto os diâmetros equatoriais maiores e com margens irregulares. Exina fina (1-3 $\mu \mathrm{m})$, reticulada heterobrocada, os retículos têm muros simples e duplicolumelados, com columelas robustas e de diferentes diâmetros; lúmens do retículo pentagonais a não-poligonais, contudo a exina forma uma calota psilado-perfurada na região equatorial aguda, apenas em Hippeastrum stylosum Herb; sexina sempre mais espessa que a nexina.

Hippeastrum stylosum Herb. apresenta a exina com um retículo heterobrocado com lúmens pentagonais a ovais (Fig. 12-13) - especialmente quando observados em M.O., cujos muros são planos mas com algumas poucas perfurações (Fig. 13). Ainda considerando a exina, deve-se ressaltar a transparência do teto observado em Hippeastrum puniceum (Lam.) Kuntze (Fig. 10) que exibe as columelas, e em alguns locais podem levar a um diagnóstico morfológico incorreto, pois essas columelas podem ser confundidas 
sob M.O. com um padrão ornamentação do tipo retículo descontínuo. Foi observada em Hippeastrum puniceum (Lam.) Kuntze uma grande quantidade de grãos de pólen com formas e tamanhos diferentes, o que pode ser explicado pela vasta ocorrência de poliploidia na espécie (Traub 1963) associada, ainda, à fragilidade da exina desses grãos de pólen que sofrem amassamentos durante os processos de acetólise e de montagem das lâminas.

Apesar de Hippeastrum Herb. ser um dos maiores gêneros da família, nenhuma contribuição sobre dados palinológicos envolvendo seus representantes foi, até então, realizada. Pode-se reservar apenas à espécietipo da família, Amaryllis belladonna L., uma breve descrição da morfologia polínica realizada por Erdtman (1966). Amaryllis, gênero mono-específico do qual Hippeastrum Herb. foi segregado, possui a ornamentação da exina bastante diferenciada do observado nas espécies aqui estudadas de Hippeastrum Herb. e segundo Erdtman (1966), esta ornamentação apresenta-se tectada com pequenos espículos diferindo, desta forma, do padrão reticulado. Tal padrão reticulado de ornamentação é muito semelhante ao observado por Meerow \& Dehgan

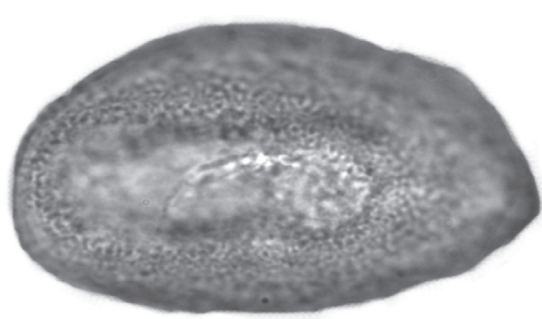

$10 \mu \mathrm{m}$
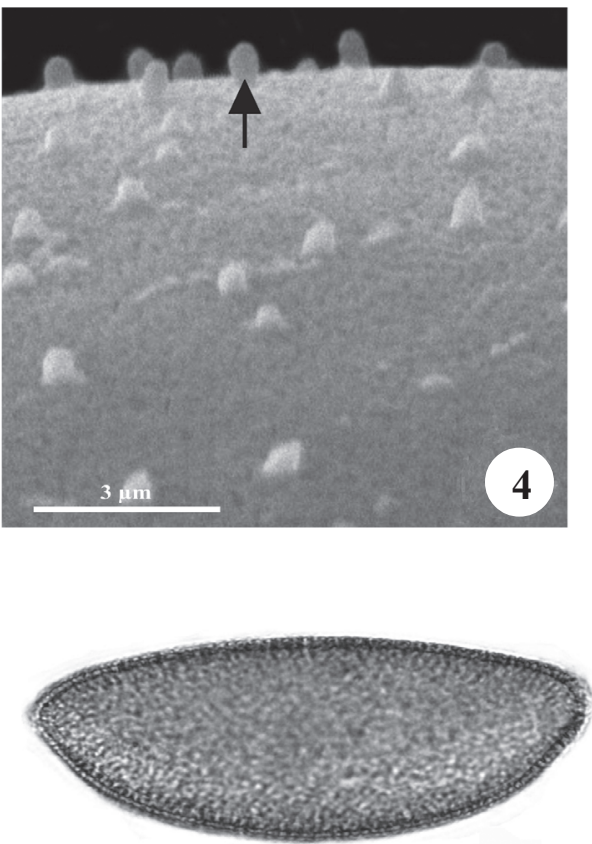

$10 \mu \mathrm{m}$

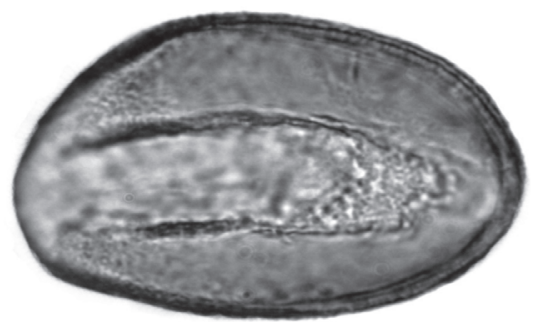

$10 \mu \mathrm{m}$
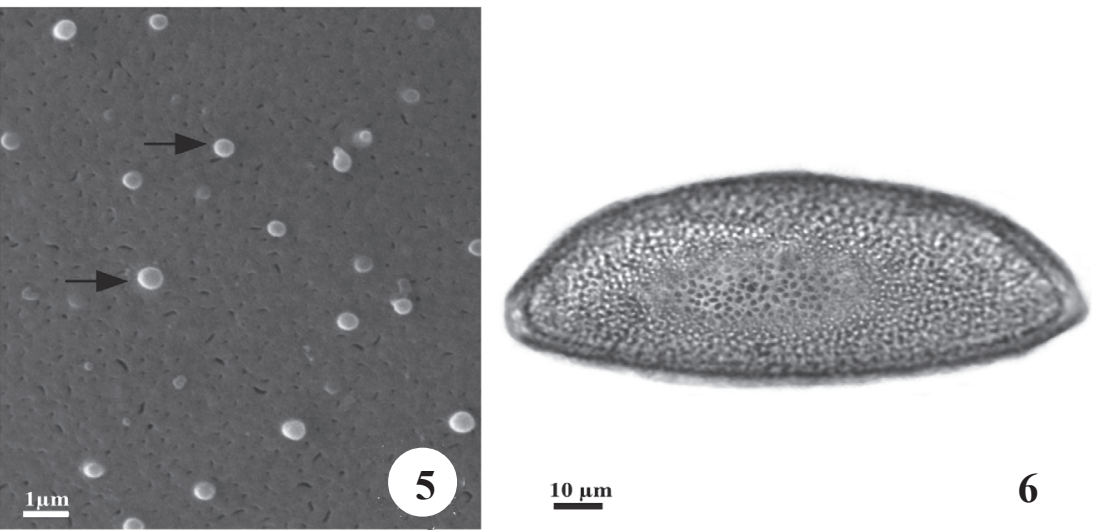

$10 \mu \mathrm{m}$
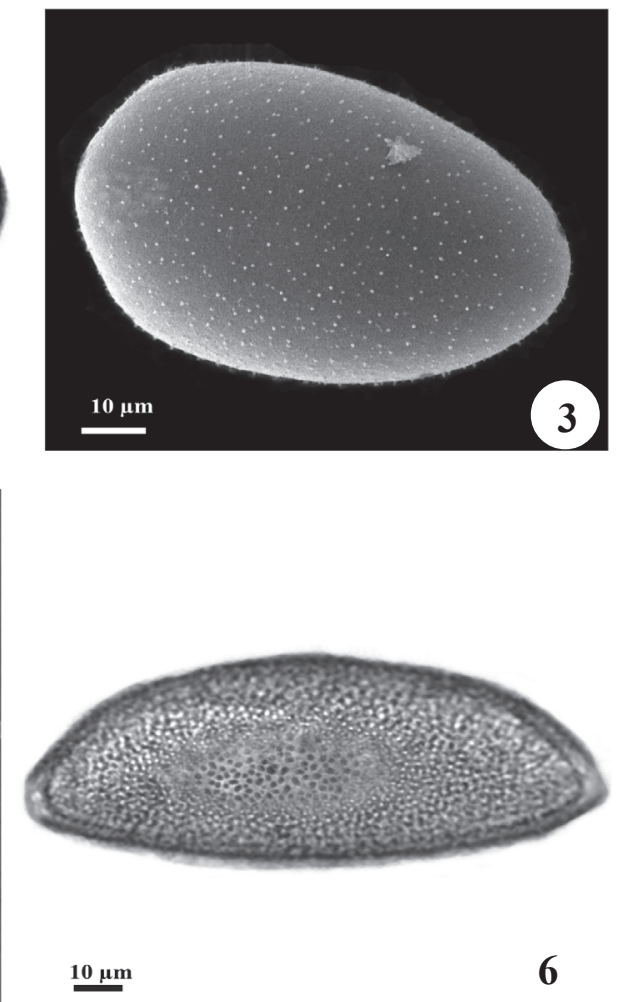

3
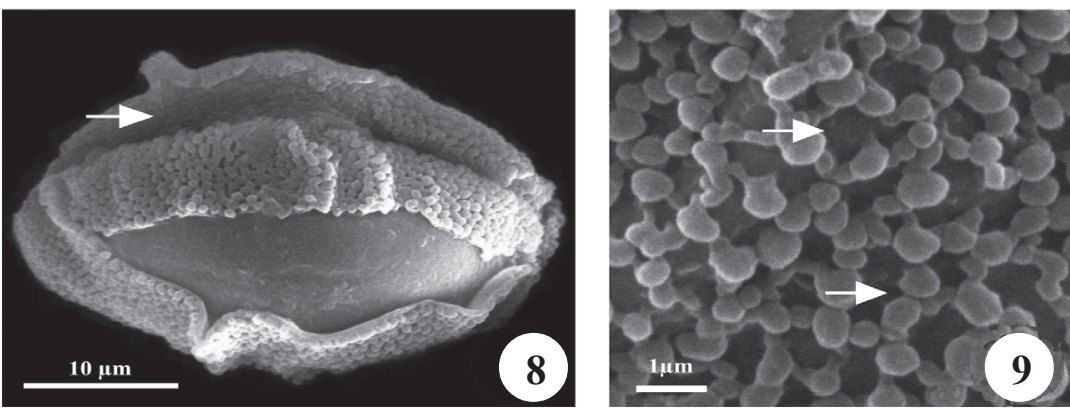

Figuras 1-9. Grãos de pólen de Amaryllidaceae s.s. 1-2. Griffinia espiritensis Ravenna var. bahiana Preuss \& Meerow: Vista polar distal, superfície e corte óptico, respectivamente. Figuras 3-5. Griffinia gardneriana (Herb.) Ravenna: 3. Vista polar proximal (MEV). 4-5. Detalhes da superfície da exina (seta $=$ processos baculiformes) (MEV). Figuras 6-7. Habranthus robustus Herb. ex Sweet: Vista equatorial, superfície e corte óptico, respectivamente. Figuras 8-9. Habranthus sylvaticus Herb.: 8. Vista equatorial, grão rompido (seta = abertura) (MEV). 9. Detalhe da superfície da exina ( $\mathrm{seta}=$ cabeça dos pilos) $(\mathrm{MEV})$. 
(1985; 1988) em algumas espécies de Hymenocallis Salisb., principalmente quando comparado com Hippeastrum stylosum Herb., devido à presença de um retículo dimórfico nas extremidades da região equatorial. Posteriormente, Meerow (1989) registrou características similares às observadas nas espécies de Hippeastrum Herb. aqui estudadas entre representantes amazônicos de Eucharis Planch. e Caliphruria Herb.

\section{Hymenocallis littoralis Salisbury}

Fig. 14-15

Grãos de pólen muito grandes (a gigantes), oblatos, amb elipsoidal. Os sulcos são tão longos quanto os diâmetros equatoriais maiores e com margens irregulares. Exina espessa (podendo alcançar ca. $11 \mu \mathrm{m})$, reticulada com abrupta mudança de padrão nas extremidades da região equatorial menor (nas calotas equatoriais) onde é clavada, lumens muito largos, interpostos entre os muros, estes últimos são sinuosos e interrompidos, teto irregular com superfície ondulada (em seção transversal), columelas compridas e muito delgadas (2-4 $\mu \mathrm{m}$ de compr.); na região equatorial menor as microclavas estão posicionadas muito próximas, que mesmo sob MEV, em baixa magnitude, parece ser uma superfície psilada; sexina mais espessa que a nexina.

A observação de microclavas (Fig. 14) nas extremidades da região equatorial de grãos de pólen de espécies de Amaryllidaceae, como a encontrada em Hymenocallis littoralis Salisb., foi referida anteriormente por Meerow \& Dehgan (1985) para Hymenocallis quitoensis Herb.; esses autores adjetivaram os grãos de pólen dessa espécie de auriculados em referência a essa diferença de ornamentação nas duas regiões equatoriais menores e agudas. Contudo, ressaltamos que o termo aurícula, criado por Potonié \& Kremp (1955), palinologicamente, segundo Punt et al. (1994), denota a presença de um espessamento radial da exina próximo às extremidades da marca de união de esporos - portanto, não aplicável aos grãos de pólen de Amaryllidaceae. Contudo, estrutura idêntica foi observada por Robyns (1963) em grãos de pólen de Bombacaceae que nominou essa região de "calota equatorial", expressão que vem sendo adotada desde então. Desta forma, sugerimos o uso dessa expressão ao termo aurícula sendo o mais adequado, que é de uso exclusivo para morfologia de esporos.

No mesmo trabalho, Meerow \& Dehgan (1985) descreveram a morfologia polínica de mais seis espécies do gênero Hymenocallis Salisb. relacionando-as com a evolução do grupo. Os autores sugerem a gradativa mudança da ornamentação da exina desde a com calotas equatoriais, passando por reticulado dimórfico até o reticulado monomórfico. A presença de calotas equatoriais nos grãos de pólen de Hymenocallis littoralis Salisb., no entanto, pode representar uma plesiomorfia dentro do grupo. Elsik (1974) menciona tal estrutura em diversos gêneros fósseis de angiospermas, principalmente não-monocotiledôneas, e em espécies ainda viventes, como representantes de Bombax spp. (Bombacaceae), como citado anteriormente. Dentre os exemplos citados pelo autor, podemos destacar os grãos de pólen do gênero fóssil Auriculiidites Elsik por compartilhar semelhanças estruturais com os de Hymenocallis littoralis Salisb., além dos exemplos citados da família Bombacaceae.

Embora Zavada (1983) tenha estudado a diversidade dos grãos de pólen entre vários representantes de monocotiledôneas, nenhuma alusão ao termo aurícula foi citada. Contraditoriamente, Elsik \& Thanikaimoni (1970) registraram uma estrutura semelhante em Bomarea lyncina Herb. (Alstroemeriaceae), sendo este o único representante do gênero, dentre as vinte espécies por eles analisadas, a possuir este caráter. Ainda assim, Meerow \& Dehgan (1985) afirmaram que as calotas equatoriais encontradas em Hymenocallis quitoensis Herb. são mais próximas, morfologicamente, aos de Bomarea lyncina Herb. do que às estruturas verificadas para Auriculiidites Elsik. Desta forma, em face da ampla distribuição deste caráter dentre as angiospermas, é possível sugerir que as calotas equatoriais possuam origem não-homóloga.

De acordo com diversos autores, exceto pela ausência de calotas equatoriais, o padrão do retículo da superfície da exina na região centro-equatorial do grão de pólen apresentado por Hymenocallis littoralis Salisb., com muros largos e lumens grandes (Fig. 15), ocorre também em Hymenocallis speciosa (Salisb.) Salisb. (Huang 1972; Erdtman 1966) e em Hymencallis declinata (Jacq.) Sweet (Erdtman 1966).

\section{Zephyranthes candida (Lindl.) Herb.}

Fig. 16-17

Grãos de pólen grandes, oblatos, amb elipsoidal. Os sulcos são tão longos quanto os diâmetros equatoriais maiores e com margens irregulares. Exina fina (ca. $1 \mu \mathrm{m})$, reticulada, heterobrocada, muros irregulares, columelas pequenas; sexina e nexina de espessura semelhante. 
Zephyranthes candida (Lindl.) Herb. possui padrão reticulado (Fig. 16, 17), contudo o retículo já está quase no limite de um microrretículo, o que aproxima essa espécie das espécies estudadas de Habranthus Herb. A descrição palinológica do apresentado corrobora o registrado por Huang (1972), no qual o autor incluiu em suas análises a espécie em questão, refletindo, desta forma, a homogeneidade das características polínicas. Padrões polínicos semelhantes foram constatados por Sharma (1967) em Zephyranthes carinata Herb. e por Rao \& Ling (1974) em Zephyranthes alba Hort.

\section{Nothoscordum pernambucanum Ravenna}

(Alliaceae s.s.)

Fig. 18-19

Grãos de pólen médios, peroblatos, amb elipsoidal. Os sulcos são tão longos quanto os diâmetros equatoriais maiores e com margens regulares. Exina fina (ca. $1 \mu \mathrm{m}$ ), teto psilado, mas apresentando pequenas depressões visíveis sob MEV que se assemelham a perfurações; columelas pequenas; sexina e nexina de espessura semelhante.

A comparação da morfologia polínica entre as espécies de Amaryllidaceae e Nothoscordum pernambucanum (Alliaceae) mostrou grande divergência quanto à ornamentação da exina e tamanho dos grãos de pólen (Tab. 1). Nothoscordum pernambucanum Rav. exibe exina tectada psilada (Fig. 18-19), característica esta compartilhada apenas com Griffinia espiritensis Rav. var. bahiana Preuss \& Meerow, porém a margem do colpo regular é um caráter único dentre as espécies analisadas (Fig. 19). Segundo Zavada (1983), a morfologia polínica aqui observada em Nothoscordum pernambucanum Rav., assim como alguns representantes de Liliaceae s.s., se enquadra no padrão evolutivo mais plesiomórfico dentre as monocotiledôneas.

Chave polínica para as espécies estudadas de Amaryllidaceae s.s. e Nothoscordum (Alliaceae s.s.) ocorrentes no Nordeste brasileiro:

1. Diâmetro equatorial maior $<50 \mu \mathrm{m}$ Nothoscordum pernambucanum Rav.

1. Diâmetro equatorial maior $\geq 50 \mu \mathrm{m}$ 2

2. Calota equatorial presente

3. Calota equatorial com superfície microclavada Hymenocallis littoralis Salisb.

3. Calota equatorial com superfície psilado-perfurada. Hippeastrum stylosum Herb.

2. Calota equatorial ausente 4

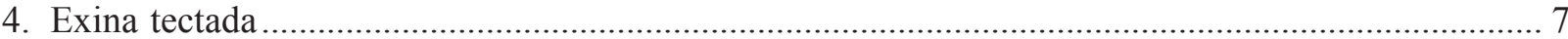

5. Diâmetro equatorial maior $<75 \mu \mathrm{m}$.... Griffinia espiritensis Rav. var. bahiana Preuss \& Meerow

5. Diâmetro equatorial maior $\geq 75 \mu \mathrm{m}$ Griffinia gardneriana (Herb.) Rav.

4. Exina semi-tectada 5

6. Razão entre o diâmetro polar e o diâmetro equatorial maior $(\mathrm{P} / \mathrm{E})>0,55$

Zephyranthes candida (Lindl.) Herb.

6. Razão entre o diâmetro polar e o diâmetro equatorial maior $\leq 0,55$ 7

7. Retículo com lumens pequenos Habranthus itaobinus Rav. Habranthus sylvaticus Herb. Habranthus robustus Herb. ex Sweet

7. Retículo com lumens amplos Hippeastrum puniceum (Lam.) Kuntze

Os grãos de pólen de Nothoscordum pernambucanum Rav. analisados mostraram-se notadamente diferentes dos grãos de pólen das espécies de Amaryllidaceae, principalmente no que se refere ao tamanho $(<50 \mu \mathrm{m})$ e até ornamentação, pois diferem daqueles das espécies estudadas de Griffinia Ker Gawl. pela presença do teto psilado - sem os processos supratectais visíveis sob MEV nos grãos de pólen do citado gênero.
$\mathrm{Na}$ chave apresentada, as espécies estudadas de Habranthus Herb. formaram um grupo estenopolínico, no qual as espécies só poderão ter seus grãos de pólen individualizados se analisados mais pormenorizadamente sob microscopia eletrônica - apesar de alguma diferença ter sido notada no tamanho dos grãos de pólen das espécies desse gênero, isso não foi considerado visto a existência de uma grande amplitude de variação nesse caráter entre indivíduos da mesma espécie. 


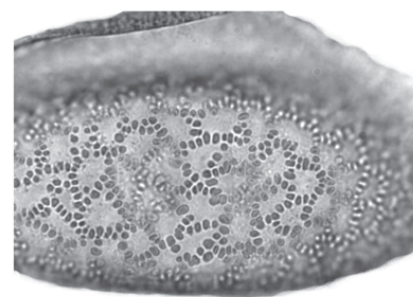

$\underline{\underline{\mu m}}$

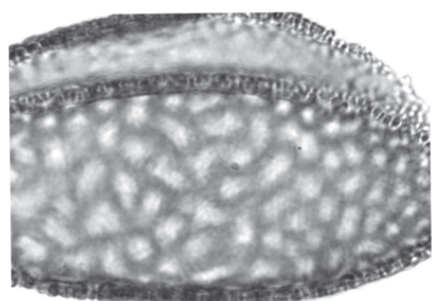

$\underline{1 \mu \mathrm{m}}$

10
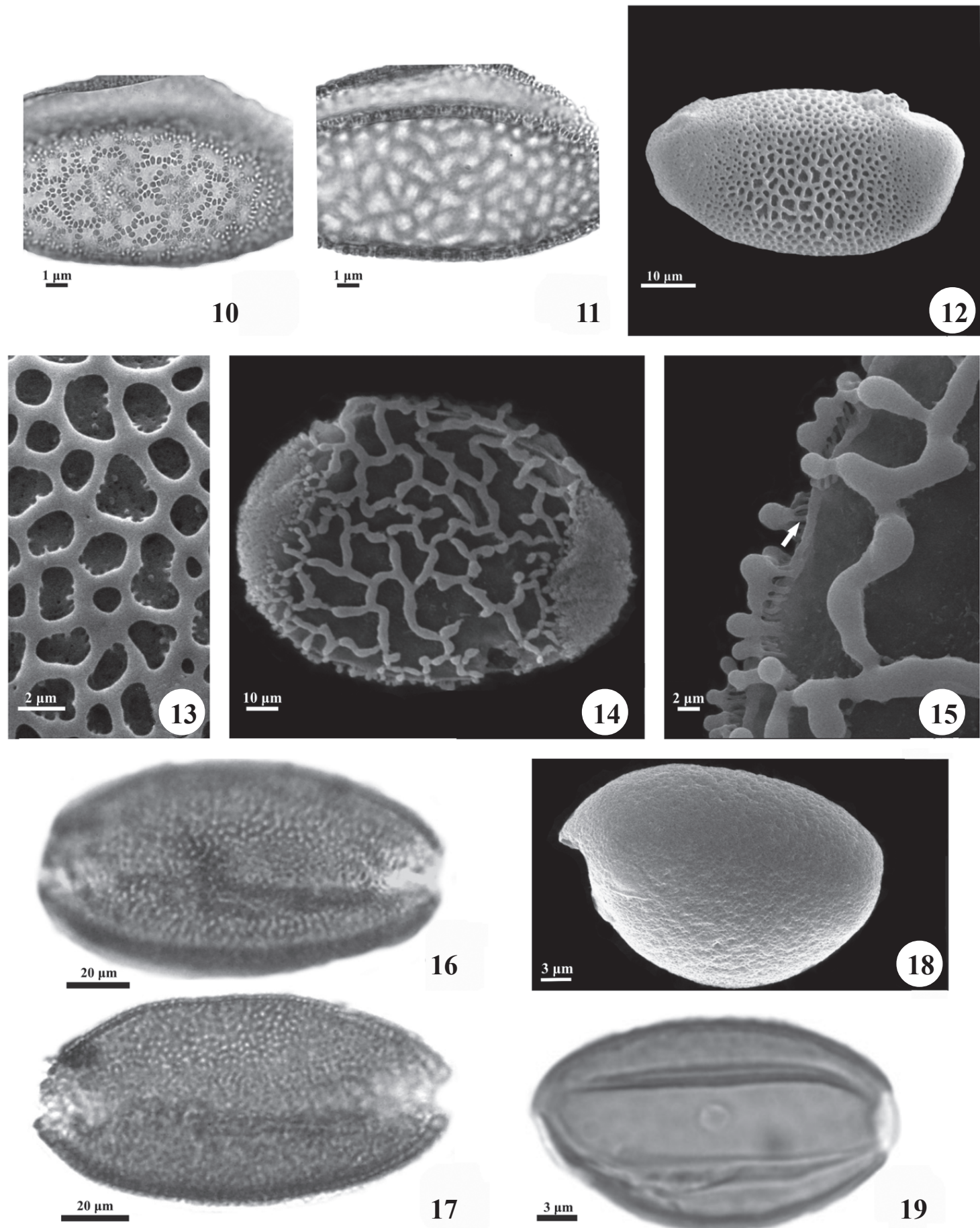

Figuras 10-19. Grãos de pólen de Amaryllidaceae s.s. e Alliaceae s.s. 10-11. Hippeastrum puniceum (Lam.) Kuntze: 10. Superfície ( 2 o foco), notar as columelas em diferentes diâmetros. 11. Vista equatorial, corte óptico. 12-13. Hippeastrum stylosum Herb.: Vista equatorial, notar as calotas equatoriais nas extremidades (MEV). 14-15. Hymenocallis littoralis Salisb.: 14. Vista polar proximal, notar calotas equatoriais nas extremidades (MEV). 15. Detalhe da superfície da exina exibindo muros irregulares e columelas muito finas (seta) (MEV). Figuras 16-17. Zephyranthes candida (Lindl.) Herb.: Vista polar distal, superfície e corte óptico, respectivamente. 18-19. Nothoscordum pernambucanum Rav.: Vista polar proximal e distal, superfície da exina (MEV) e corte óptico, respectivamente. 
Taxonomicamente, dentre os padrões evolutivos analisados em monocotiledôneas, os táxons do presente trabalho apresentaram o tipo de abertura e a forma do grão de pólen menos derivados (Walker \& Doyle 1975; Zavada 1983), muito embora, a ornamentação (micror)reticulada (exina semi-tectada) seja considerada um passo à frente do padrão psilado (exina tectada).

Segundo Erdtman (1966), algumas espécies de Amaryllidaceae exibem duas aberturas nos grãos de pólen apesar das espécies aqui estudadas serem exclusivamente monossulcadas. Duas características interessantes apresentadas nos táxons analisados são os grãos de pólen monossulcados e elíptico/elipsoidais que, segundo Zavada (1983), os mesmos constituem caracteres-base para formas mais derivadas como as inaperturadas, monoporadas e multiaperturadas exibidas por alguns grupos de monocotiledôneas como Alismataceae, Poaceae e Arecaceae, respectivamente.

Ainda no âmbito filogenético, considerando a diferença de ornamentação sobre a superfície dos grãos de pólen, a transição de um estado dimórfico (com calotas equatoriais) para um monomórfico (sem calotas equatoriais) é citada por Meerow \& Dehgan (1985) para o gênero Hymenocallis Salisb., e aqui observada nesse gênero e em Hippeastrum Herb. Essa transição constituiria um passo evolutivo no grupo das Amaryllidaceae. Essa família, segundo Walker \& Doyle (1975), juntamente com muitos dos representantes de Liliaceae s.l., compartilha características plesiomórficas retidas de ancestrais, como o gênero fóssil Liliacidites Couper.

Diante do analisado, e considerando a literatura examinada, os grãos de pólen dos táxons aqui estudados apresentaram bons caracteres que podem ser utilizados na delimitação taxonômica do grupo em questão; dentre eles são considerados a ornamentação da exina e seus arranjos e a espessura da sexina como os mais relevantes.

\section{Agradecimentos}

Ao CNPq, pela concessão de bolsa aos autores; aos Professores Dr. Marcelo Guerra e Eduardo Raduenz, pela disponibilidade de material botânico; aos amigos do Laboratório MTV/UFPE e aos integrantes do Laboratório de Micromorfologia Vegetal - LAMIV/ UEFS; aos assessores anônimos da Acta Botanica Brasilica, pelas sugestões e correções.

\section{Referências bibliográficas}

APG (Angiosperm Phylogeny Group). 2003. An update of the Angiosperm Phylogeny Group classification for the orders and families of flowering plants: APG II. Botanical Journal of the Linnean Society 141: 399-436

Elsik, W.C. 1974. Fossil auriculate pollen. Pollen et Spores 16(4): 507-533

Elsik, W.C. \& Thanikaimoni, G. 1970. Bomarea lyncina Herb. (Amaryllidaceae) and Auriculiidites Elsik. Pollen et Spores 12(12): 177-180.

Erdtman, G. 1960. The acetolysis method. A revised description. Svensk Botanisk Tidskrift 39: 561-564

Erdtman, G. 1966. Pollen morphology and plant taxonomy Angiosperms. New York, Hafner Publishing Company.

Hassal, A.H. 1842. Observations on the structure of the pollen granule, considered principally in reference to its elegibility as a means of classification. Annals and Magazine Natural History 8: 92-108.

Holmgren, P.K. \& Holmgren, N.H. 2006 onwards (continuously updated). Index Herbariorum. New York Botanical Garden. <http://sciweb.nybg.org/science2/ IndexHerbariorum.asp $>$ (Acessado em 4/julho/2006).

Huang, T.-S. 1972. Pollen flora of Taiwan. National Taiwan University Botany Department Press.

Meerow, A.W. 1989. Systematics of the Amazon lilies, Eucharis and Caliphruria (Amaryllidaceae). Annals of the Missouri Botanical Garden 76(1): 136-220.

Meerow, A.W. 2004. Amaryllidaceae. Pp. 410-412. In: N. Smith; S.A. Mori; A. Henderson; D. Wm Stevenson \& S.V. Heald (eds.). Flowering Plants of the Neotropics. New Jersey, The New York Botanical Garden.

Meerow, A.W. \& Dehgan, B. 1985. The auriculate pollen grain of Hymenocallis quitoensis Herb. (Amaryllidaceae) and its systematic implications . American Journal of Botany 72(4): 540-547.

Meerow, A.W. \& Dehgan, B. 1988. Pollen morphology of the Eucharidae (Amaryllidaceae). American Journal of Botany 75(12): 1857-1870.

Meerow, A.W.; Dehgan, N.B. \& Dehgan, B. 1986. Pollen tetrads in Stenomesson elwesii (Amaryllidaceae). American Journal of Botany 73(11): 1642-1644.

Meerow, A.W. \& Snijman, D.A. 1998. Amaryllidaceae. Pp. 83-110. In K. Kubitzki (ed.). The families and genera of vascular plants. Monocotyledons - Lilianae (except Orchidaceae). Hamburg, Germany.

Potonié, R. \& Kremp, G.O.W. 1955. Die Sporae dispersae des Ruhrkarbons, ihre Morphographie und Stratigraphie mit Ausblicken auf Arten anderer Gebiete und Zeitabschnitte. Palaeontographica, Abt. B, 98: 1-136.

Preuss, K.D. 1999. The genus Griffinia Ker Gawler (Amaryllidaceae), revisited. Herbertia 54: 51-66.

Preuss, K.D. 2004. Classification key to genus Griffinia. $<$ http://www.web.tampabay.rr.com/griffinialand/ classification.htm> (Acessado: 23/abril/2004). 
Preuss, K.D. \& Meerow, A.W. 2001. A closer look at Griffinia espiritensis Rav. (Amaryllidaceae). Herbertia 56: 119-126.

Punt, W.; Blackmore, S.; Nilsson, S. \& Le Thomas, A. 1994. Glossary of pollen and spores terminology. Utrecht, LPP Foundation.

Rao, A.N. \& Ling, L.F. 1974. Pollen morphology of certain tropical plants. Reinwardtia 9(1): 153-176.

Robyns, A. 1963. Essai de monographie du genre Bombax s.1. (Bombacaceae). Bulletin du Jardin Botanique de l'État à Bruxelles 33(1/2): 1-316.
Sharma, M. 1967. Pollen morphology of Indian monocotyledons. Journal of Palynology, Special volume: $1-98$.

Traub, H.P. 1963. Genera of the Amaryllidaceae. American Plant Life Society, California, La Jolla.

Walker, J.W. \& Doyle, J.A. 1975. The bases of angiosperm phylogeny: Palynology. Annals of the Missouri Botanical Garden 62: 664-723.

Zavada, M.S. 1983. Comparative morphology of monocot pollen and evolutionary trends of apertures and wall structures. Botanical Review 49(4): 331-379. 